\title{
Parkinson's Disease Psychosis-A Symptom Complex Signaling Risk for Increased Disability and Caregiver Burden
}

\author{
Stuart H Isaacson, MD
}

Director, Parkinson's Disease and Movement Disorders Center of Boca Raton, Boca Raton, Florida, US

\begin{abstract}
Parkinson's disease psychosis (PDP) occurs frequently in patients with Parkinson's disease (PD) and involves a spectrum of symptoms, from mild hallucinations to those that are more disruptive, as well as delusions, particularly around spousal infidelity and theft of money or possessions. Risk factors for PDP include dopaminergic (and other) medications, cognitive disorders, sleep disorders, and duration of PD. Emerging evidence suggests that PDP also reflects underlying PD pathology. Routine querying of major PDP symptoms can hasten recognition and allow earlier diagnosis of PDP. Mild hallucinations with retained insight are frequent and may be tolerable by patients and caregivers, but symptoms that can be considered as psychosis will typically increase in frequency and severity. Increasing PDP results in patient distress, increased caregiver burden, and is a leading cause of long-term nursing home placement. PDP has also been associated with an increased risk for morbidity and mortality. Ongoing research into the prevalence, risk factors, phenomenology, pathophysiology, and emerging pharmacotherapy for PDP provides hope for improved diagnosis and management of PDP.
\end{abstract}

\section{Keywords}

Parkinson's disease, psychosis, evaluation, caregiver burden, quality of life

Disclosure: Stuart H Isaacson has received honoraria for consulting and research grants from ACADIA Pharmaceuticals Inc.

Acknowledgments: Editorial assistance was provided by Catherine Amey at Touch Medical Media, London.

Open Access: This article is published under the Creative Commons Attribution Noncommercial License, which permits any noncommercial use, distribution, adaptation, and reproduction provided the original author(s) and source are given appropriate credit.

Received: February 13, 2015 Accepted: March 10, 2015 Citation: US Neurology, 2015;11(1):23-6 DOI: 10.17925/USN.2015.11.01.23

Correspondence: Stuart H Isaacson, MD, Parkinson's Disease and Movement Disorders Center of Boca Raton, Boca Raton, Florida, US. E: isaacson@ParkinsonsCenter.org

Support: The publication of this article was supported by ACADIA Pharmaceuticals Inc. The views and opinions expressed are those of the author and not necessarily those of ACADIA Pharmaceuticals Inc.

Parkinson's disease (PD) is a progressive neurodegenerative synucleinopathy. Clinical diagnosis is based on the presence of motor symptoms, including bradykinesia, rigidity, rest tremor, and postural instability. ${ }^{1}$ Although the cause of PD is still unknown, the severe nigro-striatal dopamine loss provides the basis for dopaminergic treatment of motor symptoms. Nonmotor symptoms are also prominent, probably reflecting more widespread degenerative changes in PD, and include autonomic, enteric, and neuropsychiatric symptoms. Neuropsychiatric symptoms can be prominent, such as anxiety, depression, psychosis, sleep disturbances, and cognitive impairment., ${ }^{2,3}$ This has suggested to some that PD may be accurately described as a neuropsychiatric disease rather than a pure movement disorder. ${ }^{3}$

This review aims to characterize Parkinson's disease psychosis (PDP). "Parkinson's" and "psychosis" were used as search terms in a MedLine review between the date limits of 1990 and 2014. When selecting papers for inclusion in this review, priority was given to papers of higher relevance (as determined by the MedLine database) and to more recently published papers.

\section{Prevalence and Incidence}

The prevalence of hallucinations in PD in cross-sectional prospective studies varies widely, from $16 \%$ to $75 \%$ (see Table 1). ${ }^{4-7}$ In a Norwegian population-based prevalence cohort study, 230 patients with PD were followed up prospectively for 12 years. ${ }^{8}$ The point prevalence of PDP was $17.8 \%(41 / 230)$ at baseline and increased to 48 \% (12/25) at the 12-year visit. Over the course of the study nearly two-thirds of patients (60\%, 137 patients) had developed PDP during the course of their disease. The incidence rate of PDP was 79.7 per 1,000 person-years.

The variation in the reported prevalence of PDP may be due to differences in study design and patient selection, as well as inconsistencies in screening and patient or caregiver under-reporting. ${ }^{7.9}$

- Patients may conceal their psychosis symptoms because of stigma associated with psychiatric disease, and fear of psychiatric hospitalization or long-term care placement.

- Caregivers may be unaware of mild symptoms. 


\section{Table 1: Prevalence of Hallucinations in Parkinson's Disease as Reported in Cross- sectional Prospective Studies}

\begin{tabular}{|c|c|c|}
\hline Reference & $\mathrm{n}$ & Total Prevalence (\%) \\
\hline Williams et al., $2007^{5}$ & $115^{a}$ & 75 \\
\hline Pacchetti et al., $2005^{44}$ & $289^{a}$ & 30 \\
\hline Paleacu et al., $2005^{45}$ & $276^{a}$ & 32 \\
\hline Bailbé et al., $2002^{46}$ & $152^{b}$ & 23 \\
\hline Schrag et al., $2002^{47}$ & $124^{c}$ & 23 \\
\hline Fénelon et al., 20007 & $216^{a}$ & 40 \\
\hline Aarsland et al., $1999^{4}$ & $235^{c}$ & 16 \\
\hline Inzelberg et al., $1998^{48}$ & $121^{a}$ & 37 \\
\hline Graham et al., $1997^{27}$ & $129^{a}$ & 25 \\
\hline Sanchez-Ramos et al., $1996^{18}$ & $214^{a}$ & 26 \\
\hline
\end{tabular}

Studies used a questionnaire on hallucinations, with the exception of Aarsland et al., ${ }^{4}$ in which section I of the Unified Parkinson's Disease Rating Scale was used. ${ }^{a}$ Patients from movement disorders clinics; ${ }^{b} P$ atients from hospitals or private clinics; ${ }^{c} P o p u l a t i o n-b a s e d$ study. Source: Fénelon, 2008. ${ }^{\circ}$

\section{Figure 1: Systems and Mechanisms Implicated in the Genesis of Visual Hallucinations in Parkinson's Disease}

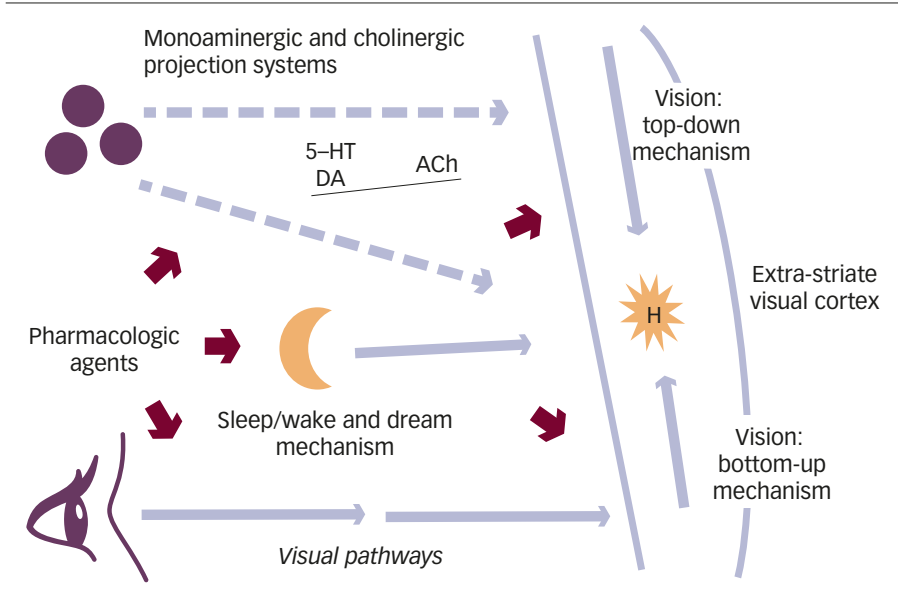

5-HT = serotonin; $A C h=$ acetylcholine; $D A=$ dopamine; $H=$ visual hallucinations . Source: Fénelon, 2008.6

- Physicians and other providers may be hesitant to query symptoms of psychosis.

- Retained insight can make PDP symptoms seem mild and nontroublesome.

- Cognitive impairment may impede evaluation of psychosis symptoms. ${ }^{6}$

For all of these reasons, PDP is probably under-recognized.? Overall, this delay in recognizing PDP can lead to symptoms becoming severe before a diagnosis of PDP is made..$^{7,10,11}$

\section{Risk Factors and Emergence of Parkinson's Disease Psychosis}

The risk for developing PDP can be influenced by both endogenous and exogenous factors. Endogenous risk factors implicated in PDP include advanced age, severity of $\mathrm{PD}$, duration of $\mathrm{PD}$, depression, cognitive impairment, and sleep disturbances.7,8,12,13 Exogenous factors may trigger PDP to emerge, or may worsen mild or subclinical PDP. These can include systemic disorders, including urinary tract (or other) infections, dehydration, fever, and medications. Both dopaminergic and anticholinergic medications increase the risk for PDP. Indeed, medications used to treat the motor systems of PD may also trigger and/or increase PDP symptoms, including levodopa:8 as PD advances levodopa dosage tends to increase, with an increased risk for PDP. Other medications commonly used to treat PD motor systems may also increase PDP, especially dopamine agonists, selegiline, amantadine, and anticholinergics. ${ }^{13}$

PDP is often thought of as a side effect of dopaminergic therapy. ${ }^{14}$ Increasing evidence, however, suggests that PDP is a manifestation of the underlying neurodegeneration of PD and is related to progression

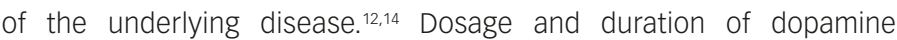
replacement therapy have not been consistently correlated with PDP. ${ }^{22,15,16}$ In an evaluation of 422 patients with PD and hallucinations, drug treatment was not identified as a risk factor for emergence of hallucinations in $\mathrm{PD}^{15}$ In addition, in a community-based study of 245 patients with $\mathrm{PD}$, anti-parkinsonian drug therapy was not shown to be associated with psychosis. ${ }^{4}$ In a study evaluating intravenous levodopa in patients with PDP, visual hallucinations did not appear to correlate with higher plasma levodopa nor to sudden changes in plasma levodopa levels. ${ }^{17} \mathrm{~A}$ proposed pathophysiologic diagram of hallucinations in PD is provided (see Figure 1).

\section{Symptoms}

Visual hallucinations are the most common symptoms of PDP. $74,18,19$ Cognitive impairment and/or dementia are independent risk factors for hallucinations, and the prevalence of hallucinations is higher in PD patients with dementia than in PD patients without dementia. $5,6,20$ PDP tends to develop in parallel with dementia, but can also herald cognitive impairment.7,8 Visual hallucinations are often preceded by sleep disturbances, vivid dreams, or REM sleep behavior disorder. ${ }^{21}$

While most hallucinations in PDP are visual, as PDP progresses, auditory, tactile, gustatory, and olfactory hallucinations can also occur. ${ }^{9,11}$ Hallucinations can occur at any time of day but are more frequent in the evening and at night. ${ }^{18}$ Older patients may be more likely to have nonvisual or mixed hallucinations than hallucinations that are purely visual in nature. ${ }^{22}$

Visual hallucinations include passage hallucinations (brief visions of a person, or more commonly an animal, passing sideways) and presence hallucinations (sensation of somebody's presence when, in fact, no person is there). ${ }^{79,14}$ More complex visual hallucinations can occur, consisting of one or more people, who may or may not be familiar, and may be living, or deceased. Also, visual hallucinations can be of animals, children, or objects. ${ }^{6}$ Illusions are misinterpretations of a real external, essentially visual stimulus, for example, seeing a tree as a person or patterns on carpets as if they are moving. 7.9,14

PDP involves a spectrum of symptoms that extends beyond formed visual hallucinations and illusions. The content of delusions is commonly paranoid, often centered on beliefs of infidelity and abandonment. 11,14,20 Delusions in patients with PDP may involve family members, which can add to the stress experienced by the family. ${ }^{11}$ Frégoli syndrome ${ }^{23}$ (a rare disorder in which a person holds a belief that different people are in fact a single person who changes their appearance or is in disguise), and Capgras syndrome ${ }^{24}$ (a delusion that someone has been replaced by an 
identical-looking impostor) have also been reported in the course of PDP, particularly in PD patients with dementia.

PDP is typically progressive over time, both in frequency and severity. Early symptoms of PDP have sometimes been considered as 'benign' since insight is often retained. However, in many patients who initially have insight into their hallucinations, insight is lost as the disease progresses. ${ }^{10}$ The likelihood that mild hallucinations will not increase in severity and/or frequency is low, frequently increasing within 2-3 years. ${ }^{10}$ Nonvisual hallucinations also occur and can complicate management. ${ }^{25}$ Even "benign," relatively minor psychotic symptoms can negatively impact quality of life ${ }^{26}$ and PD patients with hallucinations have been associated with depression and anxiety, even when insight is retained. ${ }^{7}$ As PDP progresses, delusions and paranoia can emerge, and these symptoms may become more troublesome, when accompanied by agitation. ${ }^{12,14}$

\section{Recognition and Evaluation}

Consensus diagnostic criteria for PDP have been outlined by the National Institute of Neurological Disorders and Stroke (NINDS) and the National Institutes of Mental Health (NIMH) Work Group (see Table 2). ${ }^{14}$ The NINDS$\mathrm{NIMH}$ criteria describe a chronic course of clinical features occurring with a clear sensorium and reflect the clinical, temporal, and progressive characteristics of PDP. ${ }^{26}$ According to the NIND-NIMH criteria, the diagnosis of PDP requires at least one of the following: illusions, false sense of presence, hallucinations, or delusions. These features should occur following PD onset and should be present for at least 1 month, either as recurrent or continuous symptoms, excluding other causes (dementia or other psychiatric disorders such as schizophrenia or bipolar disorder) and noting associated features, such as the presence or absence of insight, dementia, and PD treatments. ${ }^{14}$ PDP prevalence depends in part on the operational definitions employed. When the NINDS-NIMH criteria, which include minor symptoms, were applied to a cross-sectional cohort of patients with PD, the prevalence of PDP (previously defined by the presence of hallucinations and/or delusions) rose from $43 \%$ to $60 \% .{ }^{9}$ PDP does not appear to be associated with age of PD onset or dosage of dopaminergic medication. In one study, emergence of PDP occurred $>5.5$ years from PD diagnosis. ${ }^{27}$

Routine evaluation for the emergence of PDP symptoms is an important aspect of the routine care of patients with PD. , $25^{2}$ It is important to have an open and ongoing dialogue about potential PDP symptoms with the patient and their caregiver(s) and this can help to overcome the stigma associated with psychosis, 5,928 and the potential under-reporting of early PDP symptoms. Evaluation for the presence of PDP symptoms should be routinely queried at clinic visits. ${ }^{10,14,28,29}$ Common symptoms to query may include illusions; visual and other hallucinations; paranoia and other delusions; and false sense of presence or passage. If PDP is uncovered, it is important to define symptom onset, duration (recurrent or continuous for 1 month), insight, cognition, medications being taken, and to exclude other systemic causes. Initially, symptoms tend to occur intermittently with varying frequency, and can increase from several times a month to multiple times a day. PDP symptoms will often recur and/or worsen over time. The persistence and progression of symptoms thus require regular monitoring. ${ }^{14,18}$ This evaluation typically occurs in the outpatient setting, but depending on the severity of psychosis, emergency care may be necessary.

\section{Table 2: Proposed Diagnostic Criteria for Parkinson's Disease Psychosis}

1. Characteristic symptom

Presence of at least one of the following:

- Illusions

- False sense of presence

- Hallucinations

- Delusions

2. Primary diagnosis

UK brain bank criteria for PD

3. Chronology of the onset of psychosis symptoms

The symptoms in criterion 1 occur after the onset of PD

4. Duration

The symptoms(s) in criterion 1 are recurrent or continuous for 1 month

5. Exclusion of other causes

The symptoms in criterion 1 are not accounted for better by another cause of Parkinsonism such as dementia with Lewy bodies, psychiatric disorders (e.g. schizophrenia, schizoaffective disorder, or bipolar disorder), or a general mood condition including delirium

6. Associated features

- With/without insight

- With/without dementia

- With/without treatment for PD

As defined by the National Institute of Neurological Disorders and the National Institutes of Mental Health Work Group. ${ }^{14}$ PD = Parkinson's disease. Source: Ravina et al., 2007.14

\section{Caregiver Burden}

Caring for a person with PD is largely carried out by informal caregivers, such as spouses. Psychosis symptoms in PD are a strong predictor of increased caregiver burden. ${ }^{30}$ As PDP progresses, the impact on the caregiver's social activities, emotional and physical health, and overall level of stress increases. ${ }^{31}$ PDP progression and caregiver burden are both independent risk factors as well as leading causes for long-term care placement. ${ }^{32,33}$ Patients placed in a nursing home because of their PDP symptoms are likely to remain there permanently and have a higher mortality rate.32,34

A cross-sectional, population-based study in Norway was carried out in 94 caregivers of patients with PD who were living at home. ${ }^{35}$ This study identified mental symptoms as the most consistent and powerful predictors of caregiver distress, which suggests that identification and treatment of mental symptoms may reduce the distress experienced by caregivers. In a 4-year, prospective, population-based study of 178 community-dwelling individuals with PD, also conducted in Norway, the presence of hallucinations emerged as the strongest predictor of institutionalization. ${ }^{33}$

\section{Summary and Management Challenges}

PDP is a frequent but under-recognized nonmotor complication in PD, with more than $50 \%$ of patients with PD expected to develop psychosis at some time. ${ }^{8}$ This contributes significantly to diminished quality and activities of patient and caregiver daily life, and an increased risk for morbidity, and long-term care placement. Further, the presence of psychotic symptoms has been shown to be an independent predictor of mortality in PD. ${ }^{36} \mathrm{~A}$ better understanding of what causes PDP is emerging, and suggests that the effects of the underlying disease on dopaminergic, serotoninergic, and/or cholinergic pathways may be a major contributor to PDP, which can be triggered by dopaminergic medications and other 
exogenous factors. 8,9,12 Routine evaluation of PDP symptoms by treating clinicians is important to try to reduce the impact of undetected PDP on patients and their caregivers.

Once detected, PDP-management strategies should initially focus on minimizing potential offending medications and treating any systemic triggers. Approaches that reduce dopaminergic therapies though may result in suboptimal motor control, perhaps with increasing fall risk. If cognitive status is preserved, deep brain stimulation may have a role in providing motor control while allowing a reduction in medication. ${ }^{37}$

Although currently there are no FDA-approved medications for PD psychosis treatment, atypical antipsychotics are sometimes used but may worsen motor function. Physicians should also discuss PDP with patients/caregivers earlier in the course of treating PD to help improve recognition that PDP is rather common throughout the course of PD.
1. Stern MBS, A. Clinical features: motor and nonmotor. In: Schapira A, editor, Parkinson's Disease, (Oxford Neurology Library), Oxford, UK: OUP, 2010:17-25.

2. Grover S, Somaiya M, Kumar S, Avasthi A, Psychiatric aspects of Parkinson's disease, J Neurosci Rural Pract, 2015;6:65-76.

3. Weintraub D, Stern MB, Psychiatric complications in Parkinson disease, Am J Geriatr Psychiatry, 2005;13:844-51.

4. Aarsland D, Larsen JP, Cummins JL, Laake K, Prevalence and clinical correlates of psychotic symptoms in Parkinson disease: a community-based study, Arch Neurol, 1999;56:595-601.

5. Williams DR, Warren JD, Lees AJ, Using the presence of visua hallucinations to differentiate Parkinson's disease from atypica parkinsonism, J Neurol Neurosurg Psychiatry, 2008;79:652-5.

6. Fénelon G, Psychosis in Parkinson's disease: phenomenology, frequency, risk factors, and current understanding of pathophysiologic mechanisms, CNS Spectr, 2008;13 (3 Suppl. 4):18-25.

7. Fénelon $\mathrm{G}$, Mahieux F, Huon R, Ziegler M, Hallucinations in Parkinson's disease: prevalence, phenomenology and risk factors, Brain, 2000:123(Pt 4):733-45.

8. Forsaa EB, Larsen JP, Wentzel-Larsen T, et al., A 12-year population-based study of psychosis in Parkinson disease, Arch Neurol, 2010;67:996-1001

9. Fenelon $\mathrm{G}$, Soulas $\mathrm{T}$, Zenasni F, Cleret de Langavant $L$, The changing face of Parkinson's disease-associated psychosis: a cross-sectional study based on the new NINDS-NIMH criteria, Mov Disord, 2010;25:763-6.

10. Goetz CG, Fan W, Leurgans S, et al., The malignant course of "benign hallucinations" in Parkinson disease, Arch Neurol, 2006;63:713-6.

11. Goldman JG, Vaughan $C L$, Goetz $C G$, An update expert opinion on management and research strategies in Parkinson's diseas psychosis, Expert Opin Pharmacother, 2011;12:2009-24.

12. Holroyd $S$, Currie L, Wooten GF, Prospective study of hallucinations and delusions in Parkinson's disease, I Neurol Neurosurg Psychiatry 2001:70:734-8.

13. Wolters E, PD-related psychosis: pathophysiology with therapeutical strategies, J Neural Transm Suppl, 2006(71):31-7.

14. Ravina B, Marder K, Fernandez HH, et al., Diagnostic criteria for psychosis in Parkinson's disease: report of an NINDS, NIMH work group, Mov Disord, 2007:22:1061-8.

15. Merims $D$, Shabtai H, Korczyn AD, et al., Antiparkinsonian medication is not a risk factor for the development of hallucinations in Parkinson's disease, J Neural Transm, hallucinations in Parkinson's

16. Wolters EC, Psychiatric complications in the treatment of Parkinson's disease, Adv Neurol, 2001;86:385-93.
17. Goetz CG, Pappert EJ, Blasucci LM, et al., Intravenous levodopa in hallucinating Parkinson's disease patients: high-dose challenge does not precipitate hallucinations, Neurology, 1998:50:515-7.

18. Sanchez-Ramos JR, Ortoll R, Paulson GW, Visual hallucinations associated with Parkinson disease, Arch Neurol, 1996;53:1265-8.

19. Papapetropoulos S, Mash DC, Psychotic symptoms in Parkinson's disease. From description to etiology, I Neurol, 2005;252:753-64.

20. Aarsland D, Ballard C, Larsen JP, McKeith I, A comparative study of psychiatric symptoms in dementia with Lewy bodies and Parkinson's disease with and without dementia, Int I Geriatr Psychiatry, 2001;16:528-36.

21. Thanvi BR, Munshi SK, Vijaykumar N, Lo TC, Neuropsychiatric non-motor aspects of Parkinson's disease, Postgrad Med J, 2003;79:561-5.

22. Goetz CG, Wuu J, Curgian L, Leurgans S, Age-related influences on the clinical characteristics of new-onset hallucinations in Parkinson's disease patients, Mov Disord 2006:21:267-70.

23. Stewart JT, Fregoli syndrome associated with levodopa treatment, Mov Disord, 2008;23:308-9.

24. Apaydin $\mathrm{H}$, Benbir $\mathrm{G}$, Capgras syndrome during the course of Parkinson disease dementia, J Neuropsychiatry Clin Neurosci, 2014;26:E46.

25. Goetz CG, Stebbins GT, Ouyang B, Visual plus nonvisual hallucinations in Parkinson's disease: development and evolution over 10 years, Mov Disord, 2011:26:2196-200.

26. Mack J, Rabins $\mathrm{P}$, Anderson $\mathrm{K}$, et al., Prevalence of psychotic symptoms in a community-based Parkinson disease sample, Am I Geriatr Psychiatry, 2012;20:123-32.

27. Graham JM, Grunewald RA, Sagar HJ, Hallucinosis in idiopathic Parkinson's disease, J Neurol Neurosurg Psychiatry, 1997;63:434-40.

28. Fernandez $\mathrm{HH}$, Aarsland D, Fenelon $\mathrm{G}$, et al., Scales to assess psychosis in Parkinson's disease: Critique and recommendations, Mov Disord, 2008;23:484-500.

29. Voss T, Bahr D, Cummings J, et al., Performance of a shortened Scale for Assessment of Positive Symptoms for Parkinson's disease psychosis, Parkinsonism Relat Disord, 2013;19:295-9.

30. Schrag A, Hovris A, Morley D, et al., Caregiver-burden in parkinson's disease is closely associated with psychiatric symptoms, falls, and disability, Parkinsonism Relat Disord, 2006:12:35-41.

31. Aarsland D, Bronnick K, Ehrt U, et al., Neuropsychiatric symptoms in patients with Parkinson's disease and dementia: frequency, profile and associated care giver stress, J Neurol Neurosurg Psychiatry, 2007;78:36-42.
32. Goetz CG, Stebbins GT, Risk factors for nursing home placement in advanced Parkinson's disease, Neurology, 1993:43:2227-9.

33. Aarsland D, Larsen JP, Tandberg E, Laake K, Predictors of nursing home placement in Parkinson's disease: a population-based prospective study, J Am Geriatr Soc, 2000;48:938-42.

34. Goetz CG, Stebbins GT, Mortality and hallucinations in nursing home patients with advanced Parkinson's disease, Neurology, 1995;45:669-71.

35. Aarsland D, Larsen JP, Karlsen Ket al., Mental symptoms in Parkinson's disease are important contributors to caregiver distress, Int J Geriatr Psychiatry, 1999:14:866-74.

36. de Lau LM, Verbaan D, Marinus J, van Hilten JJ, Survival in Parkinson's disease. Relation with motor and non-moto features, Parkinsonism Relat Disord, 2014;20:613-6.

37. Liu Y, Li W, Tan C, et al., Meta-analysis comparing deep brain stimulation of the globus pallidus and subthalamic nucleus to treat advanced Parkinson disease, J Neurosurg, 2014;121:709-18.

38. Hasnain M, Psychosis in Parkinson's disease: therapeutic options, Drugs Today (Barc), 2011:47:353-67.

39. Fernandez $\mathrm{HH}$, Trieschmann ME, Friedman $\mathrm{JH}$, Treatment of psychosis in Parkinson's disease: safety considerations, Drug Saf, 2003:26:643-59.

40. Lutz UC, Sirfy A, Wiatr G, et al., Clozapine serum concentrations in dopamimetic psychosis in Parkinson's disease and related disorders, Eur J Clin Pharmacol, 2014;70:1471-6.

41. Hacksell U, Burstein ES, McFarland K, et al., On the discovery and development of pimavanserin: a novel drug candidate for Parkinson's psychosis, Neurochem Res, 2014:39:2008-17.

42. Cummings J. Isaacson S, Mills R Williams $H$, Chi-Burris $K$, Corbett A, et al. Pimavanserin for patients with Parkinson's disease psychosis: a randomised, placebo-controlled phase 3 trial, Lancet, 2014;383(9916):533-40.

43. Fox SH, Pimavanserin as treatment for Parkinson's disease psychosis, Lancet, 2014;383(9916):494-6.

44. Pacchetti C, Manni R, Zangaglia R, et al., Relationship between hallucinations, delusions, and rapid eye movement sleep behavior disorder in Parkinson's disease, Mov Disord, 2005:20:1439-48.

45. Paleacu D, Schechtman E, Inzelberg R, Association between family history of dementia and hallucinations in Parkinson disease, Neurology, 2005;64:1712-5.

46. Bailbe M, Karolewicz S, Neau JP, et al., [Hallucinations, delusions, and nocturnal events in 152 Parkinson's patients: a regional survey], Revue Neurologique, 2002;158:203-10.

47. Schrag A, Ben-Shlomo Y, Quinn N, How common are complications of Parkinson's disease? , J Neurol, 2002;249:419-23. 48. Inzelberg R, Kipervasser S, Korczyn AD, Auditory hallucinations in Parkinson's disease, I Neurol Neurosurg Psychiatry, 1998;64:533-5. 